\title{
Blood pressure-controlling behavior in relation to educational level and economic status among hypertensive women in Ghana
}

Bishwajit Ghose ${ }^{1}$, Sanni Yaya ${ }^{1}$

\section{Abstract}

Objective: To explore the association between economic status and educational level with self-management behavior (medication adherence, controlling body weight, reducing salt intake, performing physical exercise, reducing alcohol consumption, abstaining from smoking, increasing fruit and vegetable consumption) among hypertensive women in Ghana.

Methods: Cross-sectional data on 598 women were collected from the Ghana Demographic and Health Survey of 2014. The association between economic status and educational level with self-management behavior was measured by logistic regression methods.

Results: Mean systolic blood pressure and diastolic blood pressure were 131.4 and $86.58 \mathrm{~mm} \mathrm{Hg}$, respectively. With regard to self-management behavior, $81.8 \%$ of women reported taking medication to control high blood pressure, $44.4 \%$ reported controlling body weight, $22.5 \%$ reported reducing salt intake, $48.3 \%$ reported performing physical exercise, $74.2 \%$ reported reducing alcohol consumption, $74.2 \%$ reported abstaining from alcohol and smoking, and 20.2\% reported consuming more fruits and vegetables. Women living in the wealthiest households had significantly higher odds of maintaining healthy weight, reducing salt intake, and exercising.

Conclusion: The findings imply that socioeconomic factors may play important roles in women's adherence to blood pressure-controlling behavior. It is therefore suggested that policy makers focus on improving women's economic status as a strategy to encourage cardiovascular health-promoting behavior.

Keywords: Economic status; education; Ghana; hypertension; blood pressure management behavior; women

Significance statement: This is the first study to assess the effect of socioeconomic status on blood pressure-controlling behavior among adult women with hypertension in Ghana. The findings could be of crucial importance to health and social policy makers who are working toward reducing the burden of heart diseases and promoting healthy behavior among adult women in Ghana as well as in neighboring countries.
1. Faculty of Social Sciences, School of International Development and Global Studies, University of Ottawa, Ottawa, ON, Canada

CORRESPONDING AUTHORS: Bishwajit Ghose and Sanni Yaya Faculty of Social Sciences, School of International Development and Global Studies, University of Ottawa, 120 University Private, Ottawa, ON K1N6N5, Canada

E-mail: brammaputram@gmail. com (B. Ghose); sanni.yaya@ uottawa.ca (S. Yaya)

Received 29 November 2017; Accepted 15 June 2018

\section{Introduction}

Globally, cardiovascular diseases (CVDs) contribute to a major burden of disability and death, accounting for nearly one-third of all-cause deaths in 2008. Despite many programmatic efforts by numerous national and international organizations to combat CVDs, the prevalence has been rising at an 
alarming rate in both developed and developing countries [1]. The total number of deaths attributable to CVD increased from 14.4 million in 1990 to 17.5 million in 2005, accounting for nearly $80.0 \%$ of deaths in low-and-middle-income countries [2]. CVD is currently the largest single contributor to global mortality and is very likely to remain so through the coming decades because of the rising prevalence of the risk factors (e.g., diabetes, hypertension, obesity, physical inactivity) $[1,3,4]$. According to a study regarding the global burden of disease, the total number of hypertensive people worldwide is projected to increase by $60.0 \%$ by 2025 ( $~ 1.56$ billion) and most of the increase was predicted to occur in developing countries $[2,5]$. In the context of African countries, where the disease burden has conventionally been characterized by higher prevalence of acute-type infectious diseases (e.g., malaria), noncommunicable diseases (NCDs) such as hypertension and other CVDs are now among the leading causes of disabilities and deaths, especially among the rapidly urbanizing population across this region [2, 4-6]. For instance, in Ghana the prevalence of hypertension in urban areas was found to be more than two-fold (54.6\%) that in rural areas (19.3\%) [7].

Country-representative research evidence on CVDs and hypertension is still not available for Ghana. However, hypertension was reported to be the second leading cause of outpatient morbidity among people aged 45 years or older in the country [8], and the prevalence ranged from $19.0 \%$ to $48.0 \%$ in different studies [7]. Between 1988 and 2007, the incidence of hypertension in outpatient health facilities increased more than 10-fold, and the proportion of hypertension related to total outpatient diseases more than doubled (from 1.7\% to $4.0 \%$ ) during the same period [7]. The consequence of this rising burden of NCDs translates to enormous challenges for the underdeveloped healthcare systems and reduced national productivity in the aggregate. At the individual and household levels, CVDs are associated with significant financial burden from prolonged medication and treatment expenditures, with catastrophic consequences among the marginalized population [9, 10]. Data regarding economic analyses of CVD-related out-of-pocket expenditures are rare; however, one study conducted in Nigeria reported that hypertensive patients incurred a substantial financial burden from receiving long-term antihypertensive therapies, and the mean treatment expenditure was more than half of the citizens' monthly income [11]. While the socioeconomic consequences of CVDs seem to disproportionately affect the poor, who are also least likely to be able to afford the treatments and management, there is evidence for various intervention techniques to maintain a healthy blood pressure that could be cheaper yet highly effective methods for CVD intervention [7, 12, 13].

A growing number of studies suggest the risk of CVDs can be reduced to a great extent by control of high blood pressure (HBP), which can be achieved by lifestyle and behavioral modification techniques $[12,13]$. Behavioral interventions, in comparison with direct pharmacological intervention, refer to the adoption or modification of certain actions by individuals targeted at controlling HBP, such as reducing salt intake and abstaining from smoking and alcohol consumption [9, 10, 12]. It is difficult to monitor the potential impact of health-related behaviors on the development of CVDs as it is a very long term process. Nevertheless, epidemiological studies across countries revealed a very clear pattern regarding the intermediate physiological changes that are highly correlated with disease outcomes (e.g., elevated blood pressure) [13]. HBP is controllable to a considerable degree by maintenance of a healthy diet, exercise, adherence to drug administration, and quitting smoking and alcohol consumption. It is also reported with relation to age, body weight, dietary habit, physical activity, awareness, and areas of residency [7, 14, 15] that blood pressure control reduces mortality and produces significant cardiovascular benefits in all patients regardless of race, whereas poor blood pressure control was shown to result from lack of adherence to treatment guidelines [16, 17]. The reasons for nonadherence were reported to be associated with various demographic factors (age, area of residence), socioeconomic factors (education, income, health inequalities), and environmental factors (poor access to/availability of healthcare, poor knowledge regarding the risk factors), which can differ substantially across and within countries and social segments [9, 13, 18, 19]. However, in this line, research on the Ghanaian population was nonexistent. In this study we therefore aimed to explore the association between economic status and educational level and adherence to seven types of health-related behavior relevant to HBP among adult Ghanaian women. The results of this study are expected to contribute to the 
development of evidence-based prevention and intervention strategies across the population.

\section{Theoretical framework}

The relationship between an individual's socioeconomic status and health outcomes is explainable to a certain degree by its effect on health-related behavior. Compelling evidence suggests that educational and economic factors are strong determinants of lifestyle factors, health and disease outcomes, and overall well-being $[20,21]$. Health literacy plays a key role in the perception of health and illness, which is in turn is strongly associated with preventive/self-management behavior. Access to health information and the ability to use that information in real life as a means to promote health and avoid risky behavior are essential prerequisites for preventing NCDs such as HBP, for which self-management is a vital component of treatment [22]. Educational intervention for self-management among patients with chronic illness has been one of the most popular behavioral modification techniques. Apart from education, individual economic status is also a crucial determinant for accessing and practicing health-promoting techniques [23]. For example, households in resource-poor settings are less likely to be able to access healthy and nutritious foods and are more likely to rely on cheap sources of calories. In low-income countries such as Ghana, where health insurance is not popular, financial constraints can significantly limit people's ability to access medical care and medication. Individuals with lower socioeconomic status are also more likely to be aware of the health risks associated with a sedentary lifestyle, smoking, and drinking [21-23]. It is also worth mentioning that the association between socioeconomic status and health behavior is not linear and is assumed to be mediated by complex sociocultural constructs such as gender norms and power differentials [24, 25]. Although the effects of these constructs on health behavior are less direct, they are likely to influence behavior through perception of health and healthcare.

\section{Methods}

\section{Survey and sampling techniques}

The primary objective of the 2014 Ghana Demographic and Health Survey (DHS) was to generate recent reliable information on fertility, family planning, infant and child mortality, maternal and child health, and nutrition to assist with the making of informed policy decisions and for planning, monitoring, and evaluating programs related to health in general, and reproductive health. The survey was implemented by the Ghana Statistical Service, the Ghana Health Service, and the National Public Health Reference Laboratory of the Ghana Health Service with financial support from the United States Agency for International Development, the Global Fund through the Ghana AIDS Commission, the National Malaria Control Program, the United Nations Children's Fund (UNICEF), the United Nations Development Programme, the United Nations Population Fund (UNFPA), the International Labour Organization, the Danish International Development Agency (DANIDA), and the Government of Ghana, with technical assistance from ICF International [19].

The survey was conducted from early September to midDecember 2014. The sampling technique involved a two-stage clustering encompassing both urban and rural areas across all ten administrative regions of the country. The first stage involved the selection of clusters that are collections of enumeration areas. A total of 427 clusters were selected (216 in urban areas and 211 in rural areas). In the second stage, households were selected systematically from each enumeration area. A total of 12,831 households were selected for the survey, and 11,835 households were finally successfully interviewed, with a response rate of $99.0 \%$. Further details are provided in the Ghana DHS 2014 report [26].

\section{Variables}

The outcome variables were adherence to blood pressure management behavior of the following seven types: patients were asked if to reduce blood pressure they (1) take medicine, (2) control weight, (3) reduce salt intake, (4) perform physical exercise, (5) reduce alcohol consumption, (6) quit smoking, and (7) eat fruits and vegetables on a regular basis. Answers were coded "yes" or "no."

The explanatory variables of primary interest were economic status and educational level.

The DHS provided no direct information on personal income; however, a special technique was used to measure the wealth index of households to classify them into five groups: richest, richer, middle, poorer, and poorest. DHS programs 
used wealth index as a proxy indicator for personal income status representative of an individual's ability to meet personal healthcare needs. The process involves assigning wealth scores to household possessions (e.g., floor, wall, and roof material; type of cooking fuel; access to potable water and sanitation, ownership of radio, TV, refrigerator, motorcycle, and other items). Scoring is performed by principal component analysis, and on the basis of the weighted wealth scores, households fall into five wealth quintiles ranging from poorest to richest. Measurement of the wealth index is explained in detail elsewhere [26, 27].

On the basis of the total number of years of completion of formal education, the following categories were used for educational level: no education, primary education, secondary education, and higher education.

Age and area of residence were included in the analysis as potential confounders. Age was categorized as 15-30 years and more than 30 years, and area of residency was classified as "urban" and "rural."

\section{Data analysis}

Data were analyzed with IBM SPSS Statistics version 24. We used descriptive statistics to present respondents' demographic, economic, and blood pressure management behavioral characteristics. The Pearson $\chi^{2}$ test was used to assess the bivariate relationship of economic status and educational level with blood pressure management behaviors. We also used multiple logistic regression models for each of the behaviors to generate the exponentiated log-odds or odds ratios and corresponding $95.0 \%$ confidence intervals (CIs) to facilitate the interpretation. An odds ratio of an explanatory variable of 1 , less than 1 , and more than 1 represents absence of any effect, negative effects, and positive effects on the outcome variables, respectively. Model fitness was tested by the Hosmer and Lemeshow goodness-of-fit test. Statistical significance was set at $5.0 \%$ (two-sided).

\section{Ethics statement}

The survey was approved by the ICF International Institutional Review Board. All participants gave informed consent before interview. DHS data were accessible in the public domain in unidentifiable form, and therefore no additional approval was necessary.

\section{Results}

Population characteristics

Basic characteristics of the study population were described by the number and the corresponding percentage. Table 1 shows that most participants were older than 30 years and were urban residents. More than one-fifth of the participants had no formal education. The proportions of participants with primary, secondary, and higher education were $17.4 \%, 53.2 \%$, and $8.0 \%$, respectively.

\section{Cross tabulation}

The percentages of seven types of blood pressure management behaviors among women across the sociodemographic variables are presented in Table 2. Among the seven types of behaviors, taking medication and quitting smoking were the two most commonly used approaches to control blood pressure. Reducing the level of salt intake and regular consumption of fruits and vegetables were the least commonly followed methods. The prevalence of controlling body weight, performing physical exercise, reducing salt intake, reducing alcohol consumption, and regular fruit and vegetable consumption was

Table 1. Basic sample characteristics $(n=598)$

\begin{tabular}{lrr}
\hline Variables & Number & Percentage \\
\hline Age (years) & & \\
15-30 & 115 & 19.2 \\
$>30$ & 483 & 80.8 \\
Residency & & \\
Urban & 393 & 65.7 \\
Rural & 205 & 34.3 \\
Educational level & & \\
No education & 128 & 21.4 \\
Primary education & 104 & 17.4 \\
Secondary education & 318 & 53.2 \\
Higher education & 48 & 8.0 \\
Economic status & & \\
Poorest & 70 & 11.7 \\
Poorer & 78 & 13.0 \\
Middle & 132 & 22.1 \\
Richer & 141 & 23.6 \\
Richest & 177 & 29.6 \\
\hline
\end{tabular}

Data from Ghana Demographic and Health Survey, 2014. 
Table 2. Adherence (as a percentage) to blood pressure management behaviors across sociodemographic variables

\begin{tabular}{|c|c|c|c|c|c|c|c|}
\hline \multirow[t]{2}{*}{$\begin{array}{l}\text { All } \\
\text { participants }\end{array}$} & $\begin{array}{l}\text { Medication } \\
\text { adherence }\end{array}$ & $\begin{array}{r}\text { Control } \\
\text { body weight }\end{array}$ & $\begin{array}{r}\text { Reduce } \\
\text { salt intake }\end{array}$ & $\begin{array}{r}\text { Perform physical } \\
\text { exercise }\end{array}$ & $\begin{array}{r}\text { Stop } \\
\text { smoking }\end{array}$ & $\begin{array}{l}\text { Stop alcohol } \\
\text { consumption }\end{array}$ & $\begin{array}{l}\text { Consume fruits } \\
\text { and vegetables }\end{array}$ \\
\hline & 81.8 & 44.4 & 22.5 & 48.3 & 74.2 & 74.2 & 20.2 \\
\hline \multicolumn{8}{|l|}{ Age (years) } \\
\hline $36-45$ & 80.9 & 44 & 20.6 & 48.3 & 71.3 & 81.8 & 18.2 \\
\hline$>45$ & 83.7 & 50 & 24 & 51 & 76 & 85.6 & 19.2 \\
\hline$P$ & 0.082 & 0.003 & 0.046 & 0.212 & 0.276 & 0.258 & 0.502 \\
\hline \multicolumn{8}{|l|}{ Residency } \\
\hline Urban & 82.1 & 42 & 21.5 & 45 & 74.6 & 82.7 & 19.2 \\
\hline Rural & 81.1 & 49.1 & 24.5 & 54.7 & 73.6 & 86.8 & 22 \\
\hline$P$ & 0.378 & 0.018 & 0.076 & 0.013 & 0.538 & 0.013 & 0.02 \\
\hline \multicolumn{8}{|l|}{ Educational level } \\
\hline No education & 84 & 50 & 20 & 43 & 87 & 89 & 23 \\
\hline \multicolumn{7}{|l|}{ education } & 21.3 \\
\hline $\begin{array}{l}\text { Secondary } \\
\text { education }\end{array}$ & 81.4 & 41.3 & 21.9 & 48.6 & 78.7 & 79.3 & 17.4 \\
\hline \multicolumn{7}{|l|}{ education } & 28.6 \\
\hline$P$ & 0.254 & 0.185 & 0.576 & 0.003 & 0.02 & 0.086 & 0.053 \\
\hline \multicolumn{8}{|l|}{ Economic status } \\
\hline Poorest & 78.5 & 39.7 & 22.4 & 44.7 & 69.4 & 85.7 & 18.4 \\
\hline Poorer & 85.1 & 39.3 & 17.9 & 50.7 & 80.6 & 88.1 & 26.9 \\
\hline Middle & 83.3 & 43.1 & 24.5 & 52 & 74.5 & 79.4 & 18.6 \\
\hline Richer & 81.8 & 43.3 & 20.6 & 35.5 & 69.2 & 82.2 & 22 \\
\hline Richest & 81.4 & 73.5 & 24.8 & 75.5 & 76.6 & 86.5 & 15.9 \\
\hline$P$ & 0.941 & $<0.001$ & 0.02 & $<0.001$ & 0.664 & 0.438 & 0.15 \\
\hline
\end{tabular}

Data from [26].

$44.4 \%(95.0 \%$ CI $43.81-44.99 \%), 48.3 \%(95.0 \%$ CI $47.34-$ $49.26 \%), 22.5 \%(95.0 \%$ CI $21.91-23.09 \%), 74.2 \%(95.0 \%$ CI $73.24-75.16 \%)$, and $20.2 \%(95.0 \%$ CI $19.24-21.16 \%)$, respectively.

The $\chi^{2}$ test showed that participants in the youngest age group were most likely to have lower alcohol consumption, be nonsmokers, and regularly consume fruits and vegetables; the participants who showed the highest rates of adherence to regular medication, controlling body weight, reducing salt intake, and exercising were those aged more than 45 years. Except for medication adherence and stopping smoking, rural participants had a higher likelihood of maintaining blood pressure management behaviors than urban participants. Participants from richer or the richest households and who had higher than secondary level education were also more likely to adhere to all blood pressure management behaviors.

The results of the multivariable analysis regarding the association between the seven types of blood pressure management behaviors with economic status and educational level are presented in Table 3. The results indicate that compared with participants from the poorest households, those from richer or the richest households had higher odds of practicing all the self-management techniques. However, the associations were significant for weight control, reducing salt intake, 


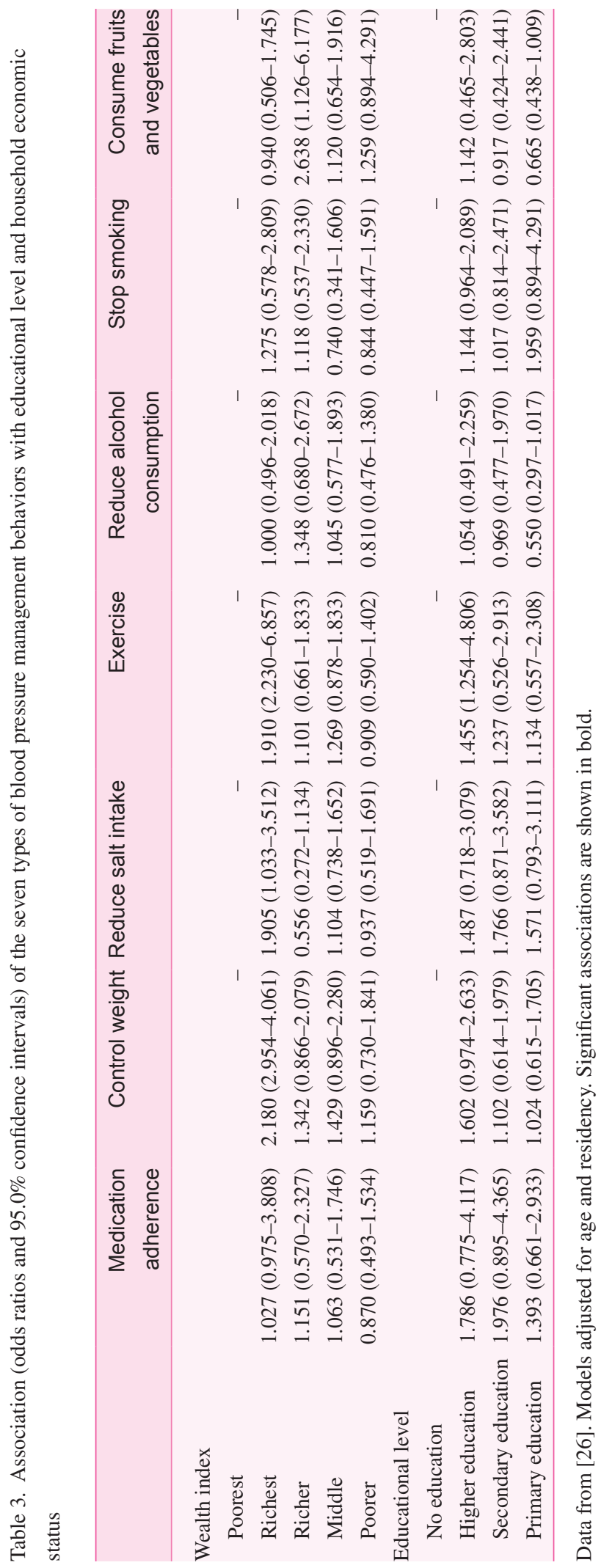

and exercising only. Compared with women from the poorest households, the odds of controlling body weight (adjusted odds ratio 2.2, 95.0\% CI 2.954-4.061), reducing salt intake (adjusted odds ratio 1.9, 95.0\% CI 1.033-3.512), and exercising regularly (adjusted odds ratio $1.9 ; 95.0 \%$ CI 2.230-6.857) were about two-fold higher among women from the richest households. Economic status did not show any significant association with medication adherence, reducing alcohol consumption, and consumption of fruits and vegetables.

Regarding educational level, on the other hand, the odds of medication adherence, controlling weight, and reducing salt intake for women with higher education were $78.0 \%$ (95.0\% CI $0.775-4.117), 60.0 \%$ (95.0\% CI $0.974-2.633$ ), and $48.0 \%$ (95.0\% CI 0.738-1.652), respectively. However, only with physical exercise was the association statistically significant. Women with higher education were $45.0 \%$ (95.0\% CI 1.254 4.806) more likely to perform physical exercise compared with those who had no education.

\section{Discussion}

In the present study, we focused on the impact of economic status and educational level on blood pressure-controlling behavior among hypertensive women in Ghana. The study results revealed that both educational level and economic status were significantly associated with adherence to blood pressure-controlling behaviors in the sample population. Financial solvency and education were proved to act as enabling factors that help change subjective norms and behavior associated with improved cardiovascular outcomes [28, 29]. With emerging evidence on the importance of behavioral interventions for NCDs, there has been increasing research interest in identifying the underlying factors that influence adherence to healthy behaviors among NCD patients. Evidence from the African context is rare; however, studies from other developing countries showed that lack of awareness regarding the risk factors for $\mathrm{HBP}$ and poor adherence to medication and HBP-controlling behaviors pose serious challenges for healthcare providers, and hence routinely encourage self-management of blood pressure [17, 28, 30].

In line with previous findings, our results showed that educational level was positively associated with medication adherence and abstinence from tobacco and alcohol [31-33]. Higher education and higher income were proved to promote physical 
activity through self-efficacy for exercise, healthier influences from social network members, and more access to resources that facilitate physical activity $[34,35]$. A case-control study among Korean adults on a medication self-management education program found that education had a positive effect on improving medication awareness and decreasing medication misuse behaviors, and the study regarded an education program as an effective intervention for improving self-management for the hypertensive elderly population [36]. Although provider education for controlling HBP is a commonly used technique, the importance of educating patients is also becoming evident from various studies. A cluster randomized trial involving two hospital-based and eight community-based clinics in the United States reported that patients in the group where both the provider and the patients received educational materials had better blood pressure-controlling outcomes compared with the provider-education-only group [37]. In addition, economic status was also found to be a promoting factor for adherence to self-management techniques. This finding is consistent with the findings of Grimm et al. [38] and Middaugh et al. [39], who found a direct link between poverty and lower consumption of fruits and vegetables $[38,40]$. Lower socioeconomic status can lead to adoption of an unhealthy lifestyle, such as increased level of alcohol consumption and smoking with a poor diet and less frequent physical exercise, which can worsen blood pressure outcomes [40, 41].

Self-management of chronic diseases is a complex process, as once diagnosed they can require lifelong therapy and monitoring. Patients, caregivers, and physicians need to make constant efforts with regard to medication, diet control, and physical exercise adherence, while all the effects with regard to the clinical outcomes are referred to as self-management [38]. Patient education and provider education constitute the most crucial components of effective blood pressure control. However, patient education plays a more prominent role in ensuring long-term improvement in cardiovascular outcomes, since the process requires a range of behavioral modifications that may not be achieved by the provider-side training alone. Therefore the healthcare system is required to take responsibility for raising awareness regarding the disease and associated risk factors to enhance self-management by positive behavioral change among CVD patients [38]. This can be achieved by population-based epidemiological studies exploring various enabling and limiting factors of self-management. Development of a good understanding of these factors can help healthcare systems devise effective intervention tools. In addition to patient-side education, it is of particular importance to address health expenditure-related issues that may constrain patients' ability to adopt healthy lifestyles.

To our knowledge, this is the first study to focus on blood pressure management behaviors in a Ghanaian population. The findings of the study can provide valuable insights to formulate evidence-based policy making targeted at promoting blood pressure management behaviors among adult women in Ghana. However, the findings need to be interpreted with some important limitations in mind. As the data were crosssectional, the study does not allow causal inference from the findings. Blood pressure management behaviors were selfreported, and therefore the study remains subject to recall bias and overreporting/underreporting.

\section{Conclusion}

The findings suggested that educational and economic constraints pose significant barriers to adhering to blood pressure management behaviors among women in Ghana. Although the study was cross-sectional and cannot claim any causal relationship, the findings provide some indication that addressing socioeconomic disparities may promote healthy behaviors, including adherence to medication and abstaining from risk factors such as smoking and alcohol drinking. In this regard, community-oriented health literacy and behavioral modification programs may serve as effective approaches for promoting self-management of HBP. We also recognize that there remains a lack of quality evidence on self-management behaviors among Ghanaian women. This study thus sets the ground for further studies along this line and suggests a wider variety of blood pressure-controlling behaviors and socioeconomic indicators should be explored.

\section{Conflict of interest}

The authors declare that they have no conflicts of interest.

\section{Funding}

No funding was available for this study. 


\section{Author contributions}

The study was conceived by GB. Data collection and analysis were conducted by GB. SY contributed to the interpretation of the findings and drafting of the manuscript. Both authors critically revised the final version and approved it for publication.

\section{References}

1. Deaton C, Froelicher ES, Wu LH, Ho C, Shishani K, Jaarsma T. The global burden of cardiovascular disease. Eur J Cardiovasc Nurs 2011;10(Suppl 2):S5-13.

2. Kearney PM, Whelton M, Reynolds K, Muntner P, Whelton PK, He J. Global burden of hypertension: analysis of worldwide data. Lancet 2005;365(9455):217-23.

3. Chockalingam A, Campbell NR, Fodor JG. Worldwide epidemic of hypertension. Can J Cardiol 2006;22(7):553-5.

4. Meinema JG, van Dijk N, Beune EJAJ, Jaarsma DADC, van Weert HCPM, Haafkens JA. Determinants of adherence to treatment in hypertensive patients of African descent and the role of culturally appropriate education. PLoS One 2015;10(8):e0133560.

5. Bosworth HB, Dudley T, Olsen MK, Voils CI, Powers B, Goldstein MK, et al. Racial differences in blood pressure control: potential explanatory factors. Am J Med 2006;119(1):70.e9-15.

6. Lawes CM, Vander Hoorn S, Law MR, Elliott P, MacMahon S, Rodgers AJ. Blood pressure and the global burden of disease 2000. Part 1: estimates of blood pressure levels. J Hypertens 2006;24(3):413-22.

7. Bosu WK. Epidemic of hypertension in Ghana: a systematic review. BMC Public Health 2010;10:418.

8. Ministry of Health. The Ghana Health Sector 2006 Programme of Work. 2005.

9. Elliott WJ. The economic impact of hypertension. J Clin Hypertens (Greenwich) 2003;5(3Suppl 2):3-13.

10. Flack JM, Casciano R, Casciano J, Doyle J, Arikian S, Tang S, et al. Cardiovascular disease costs associated with uncontrolled hypertension. Manag Care Interface 2002;15(11):28-36.

11. Ilesanmi OS, Ige OK, Adebiyi AO. The managed hypertensive: the costs of blood pressure control in a Nigerian town. Pan Afr Med J 2012;12:96.

12. Wright JT Jr, Dunn JK, Cutler JA, Davis BR, Cushman WC, Ford $\mathrm{CE}$, et al. Outcomes in hypertensive black and nonblack patients treated with chlorthalidone, amlodipine, and lisinopril. J Am Med Assoc 2005;293(13):1595-608.

13. Ogedegbe G. Barriers to optimal hypertension control. J Clin Hypertens (Greenwich) 2008;10(8):644-6.
14. Duda RB, Kim MP, Darko R, Adanu RM, Seffah J, Anarfi JK, et al. Results of the Women's Health Study of Accra: assessment of blood pressure in urban women. Int J Cardiol 2007;117:115-22.

15. Addo J, Amoah AG, Koram KA. The changing patterns of hypertension in Ghana: a study of four rural communities in the Ga District. Ethn Dis 2006;16(4):894-9.

16. Conn VS, Ruppar TM, Chase JA, Enriquez M, Cooper PS. Interventions to improve medication adherence in hypertensive patients: systematic review and meta-analysis. Curr Hypertens Rep 2015;17(12):94.

17. Bosworth HB, Olsen MK, Grubber JM, Neary AM, Orr MM, Powers BJ, et al. Two self-management interventions to improve hypertension control: a randomized trial. Ann Intern Med 2009;151(10):687-95.

18. Hunte HER, Mentz G, House JS, Schulz AJ, Williams DR, Elliott $\mathrm{MR}$, et al. Variations in hypertension-related outcomes among blacks, whites and Hispanics in two large urban areas and in the United States. Ethn Dis 2012;22(4):391-7.

19. Meshram II, Rao MVV, Rao VS, Laxmaiah A, Polasa K. Regional variation in the prevalence of overweight/obesity, hypertension and diabetes and their correlates among the adult rural population in India. Br J Nutr 2016;115(7):1265-72.

20. Nandi A, Glymour MM, Subramanian SV. Association among socioeconomic status, health behaviors, and all-cause mortality in the United States. Epidemiology 2014;25(2):170-7.

21. Race, socioeconomic status, and risk of a catastrophic health condition in later life. Res Sociol Health Care 2000;18:151-65.

22. Stringhini S, Dugravot A, Shipley M, Goldberg M, Zins M, Kivimäki $\mathrm{M}$, et al. Health behaviours, socioeconomic status, and mortality: further analyses of the British Whitehall II and the French GAZEL prospective cohorts. PLoS Med 2011;8(2):e1000419.

23. Hanson MD, Chen E. Socioeconomic status and health behaviors in adolescence: a review of the literature. J Behav Med 2007;30(3):263-85

24. Fiaveh DY, Izugbara CO, Okyerefo MPK, Reysoo F, Fayorsey CK. Constructions of masculinity and femininity and sexual risk negotiation practices among women in urban Ghana. Cult Health Sex 2015;17(5):650-62.

25. Fiaveh DY, Okyerefo MPK, Fayorsey CK. Women's experiences of sexual pleasure in Ghana. Sex Cult 2015;19(4):697-714.

26. Ghana Statistical Service, Ghana Health Service, ICF International. Ghana Demographic and Health Survey 2014. Rockville: Ghana Statistical Service, Ghana Health Service, and ICF International; 2015. 
27. Yaya S, Bishwajit G, Danhoundo G, Shah V, Ekholuenetale M. Trends and determinants of HIV/AIDS knowledge among women in Bangladesh. BMC Public Health 2016;16:812.

28. Maimaris W, Paty J, Perel P, Legido-Quigley H, Balabanova D, Nieuwlaat R, et al. The influence of health systems on hypertension awareness, treatment, and control: a systematic literature review. PLoS Med 2013;10(7):e1001490.

29. Flynn SJ, Ameling JM, Hill-Briggs F, Wolff JL, Bone LR, Levine DM, et al. Facilitators and barriers to hypertension self-management in urban African Americans: perspectives of patients and family members. Patient Prefer Adherence 2013;7:741-9.

30. Staessen JA, Den Hond E, Celis H, Fagard R, Keary L, Vandenhoven G, et al. Antihypertensive treatment based on blood pressure measurement at home or in the physician's office: a randomized controlled trial. J Am Med Assoc 2004;291(8): 955-64.

31. Gaziano TA, Bertram M, Tollman SM, Hofman KJ. Hypertension education and adherence in South Africa: a cost-effectiveness analysis of community health workers. BMC Public Health 2014;14:240.

32. Crone M, Reijneveld S, Willemsen M, van Leerdam FJM, Spruijt $\mathrm{R}$, Sing R. Prevention of smoking in adolescents with lower education: a school based intervention study. J Epidemiol Community Health 2003;57(9):675-80.

33. Zebregs S, van den Putte B, de Graaf A, Lammers J, Neijens $P$. The effects of narrative versus non-narrative information in school health education about alcohol drinking for low educated adolescents. BMC Public Health 2015;15:1085.
34. McAuley E, Konopack JF, Morris KS, Motl RW, Hu L, Doerksen SE, et al. Physical activity and functional limitations in older women: influence of self-efficacy. Gerontol B Psychol Sci Soc Sci 2006;61(5):P270-7.

35. Chad KE, Reeder BA, Harrison EL, Ashworth NL, Sheppard SM, Schultz SL, et al. Profile of physical activity levels in communitydwelling older adults. Med Sci Sports Exerc 2005;37(10):1774-84.

36. Lee JK. Evaluation of a medication self-management education program for elders with hypertension living in the community. J Korean Acad Nurs 2013;43(2):267-75.

37. Roumie CL, Elasy TA, Greevy R, Griffin MR, Liu X, Stone WJ, et al. Improving blood pressure control through provider education, provider alerts, and patient education: a cluster randomized trial. Ann Intern Med 2006;145(3):165-75.

38. Grimm KA, Foltz JL, Blanck HM, Scanlon KS. Household income disparities in fruit and vegetable consumption by state and territory: results of the 2009 Behavioral Risk Factor Surveillance System. J Acad Nutr Diet 2012;112(12):2014-21.

39. Middaugh AL, Fisk PS, Brunt A, Rhee YS. Few associations between income and fruit and vegetable consumption. J Nutr Educ Behav 2012;44(3):1.

40. de Silva V, Samarasinghe D, Hanwella R. Association between concurrent alcohol and tobacco use and poverty. Drug Alcohol Rev 2011;30(1):69-73.

41. Cohen DA, Han B, Derose KP, Williamson S, Marsh T, Rudick $\mathrm{J}$, et al. Neighborhood poverty, park use, and park-based physical activity in a southern California city. Soc Sci Med 2012;75(12):2317-25 